\title{
Group Signatures with Almost-for-Free Revocation
}

\author{
Benoît Libert ${ }^{1, \star}$, Thomas Peters ${ }^{1, \star \star}$, and Moti Yung ${ }^{2}$ \\ 1 Université Catholique de Louvain, ICTEAM Institute, Belgium \\ 2 Google Inc. and Columbia University, USA
}

\begin{abstract}
Group signatures are a central cryptographic primitive where users can anonymously and accountably sign messages in the name of a group they belong to. Several efficient constructions with security proofs in the standard model (i.e., without the random oracle idealization) appeared in the recent years. However, like standard PKIs, group signatures need an efficient revocation system to be practical. Despite years of research, membership revocation remains a non-trivial problem: many existing solutions do not scale well due to either high overhead or constraining operational requirements (like the need for all users to update their keys after each revocation). Only recently, Libert, Peters and Yung (Eurocrypt'12) suggested a new scalable revocation method, based on the Naor-Naor-Lotspiech (NNL) broadcast encryption framework, that interacts nicely with techniques for building group signatures in the standard model. While promising, their mechanism introduces important storage requirements at group members. Namely, membership certificates, which used to have constant size in existing standard model constructions, now have polylog size in the maximal cardinality of the group (NNL, after all, is a tree-based technique and such dependency is naturally expected). In this paper we show how to obtain private keys of constant size. To this end, we introduce a new technique to leverage the NNL subset cover framework in the context of group signatures but, perhaps surprisingly, without logarithmic relationship between the size of private keys and the group cardinality. Namely, we provide a way for users to efficiently prove their membership of one of the generic subsets in the NNL subset cover framework. This technique makes our revocable group signatures competitive with ordinary group signatures (i.e., without revocation) in the standard model. Moreover, unrevoked members (as in PKIs) still do not need to update their keys at each revocation.
\end{abstract}

\section{Introduction}

Group signatures, as suggested by Chaum and van Heyst 29], allow members of a group managed by some authority to sign messages in the name of the group

\footnotetext{
* This author acknowledges the Belgian Fund for Scientific Research (F.R.S.-F.N.R.S.) for his "Collaborateur scientifique" fellowship.

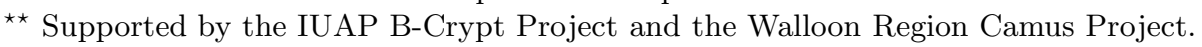


while hiding their identity. At the same time, a tracing authority can identify the signer if necessary. A crucial problem is the revocation of the anonymous signing capability of users who leave (or are banned from) the group.

\subsection{Related Work}

ORDINARY GROUP SIGNATURES. The first efficient and provably coalition-resistant group signature dates back to the work of Ateniese, Camenisch, Joye and Tsudik 6]. By the time their scheme appeared, the security of the primitive was not appropriately formalized yet. Suitable security definitions remained lacking until the work of Bellare, Micciancio and Warinschi [8] (BMW) who captured all the requirements of group signatures in three properties. In (a variant of) this model, Boneh, Boyen and Shacham [14] obtained very short signatures using the random oracle methodology 9 .

The BMW model assumes static groups where no new member can be introduced after the setup phase. The setting of dynamically changing groups was analyzed later on by Bellare-Shi-Zhang [10] and, independently, by Kiayias and Yung [40]. In the models of [1040], constructions featuring relatively short signatures were proposed in 4930. A construction in the standard model was also suggested by Ateniese et al. [5] under interactive assumptions. At the same time, Boyen and Waters gave a different solution [18] without random oracles using more standard assumptions. By improving upon their own scheme, they managed [19 to obtain signatures of constant size. Their constructions [1819] were both presented in the BMW model [8] and provide anonymity in the absence of signature opening oracle. In the dynamic model [10], Groth [34] showed a system in the standard model with $O(1)$-size signatures but, due to very large hidden constants, his scheme was mostly a feasibility result. Later on, Groth came up with an efficient realization [35] (and signatures of about 50 group elements) with the strongest anonymity level.

Revocation. As in ordinary PKIs, where certificate revocation is a critical issue, membership revocation is a complex problem that has been extensively studied [20 7 2617] in the last decade. Generating a new group public key and distributing new signing keys to unrevoked members is a simple solution. In large groups, it is impractical to update the public key and provide members with new keys after they joined the group. Bresson and Stern suggested a different approach 20] consisting of having the signer prove that his membership certificate does not belong to a list of revoked certificates. Unfortunately, the length of signatures grows with the number of revoked members. In forwardsecure group signatures, Song [50] chose a different way to handle revocation but verification takes linear time in the number of excluded users.

Camenisch and Lysyanskaya [26] proposed an elegant method using accumulator:11 11. Their technique, also used in [52|24, allows revoking members while

\footnotetext{
${ }^{1}$ An accumulator is a kind of "hash" function mapping a set of values to a short, constant-size string while allowing to efficiently prove that a specific value was accumulated.
} 
keeping $O(1)$ costs for signing and verifying. The downside of this approach is its history-dependence: it requires users to follow the dynamic evolution of the group and keep track of all changes: each revocation incurs a modification of the accumulator value, so that unrevoked users have to upgrade their membership certificate before signing new messages. In the worst case, this may require up to $O(r)$ exponentiations, if $r$ is the number of revoked users.

Another drawback of accumulator-based approaches is their limited applicability in the standard model. Indeed, for compatibility reasons with the central tool of Groth-Sahai proofs, pairing-based accumulators are the only suitable candidates. However, in known pairing-based accumulators [424, public keys have linear size in the maximal number of accumulations, which would result in linearsize group public keys in immediate implementations. To address this concern in delegatable anonymous credentials, Acar and Nguyen [4 chose to sacrifice the constant size of proofs of non-membership but, in group signatures, this would prevent signatures from having constant size. Boneh, Boyen and Shacham 14 managed to avoid linear dependencies in a revocation mechanism along the lines of [26. Unfortunately, their technique does not seem to readily interact 2 with Groth-Sahai proofs 36] so as to work in the standard model.

In [21, Brickell considered the notion of verifier-local revocation group signatures, for which formal definitions were given by Boneh and Shacham [17] and other extensions were proposed in 465342. In this approach, revocation messages are only sent to verifiers and the signing algorithm is completely independent of the number of revocations. Verifiers take as additional input a revocation list (RL), maintained by the group manager, and have to perform a revocation test for each $\mathrm{RL}$ entry in order to be convinced that signatures were not issued by a revoked member (a similar revocation mechanism is used in [22]). The verification cost is thus inevitably linear in the number of expelled users.

In 2009, Nakanishi, Fuji, Hira and Funabiki 45] came up with a revocable group signature with constant complexities for signing/verifying. At the same time, group members never have to update their keys. On the other hand, their proposal suffers from linear-size group public keys in the maximal number $N$ of users, although a variant reduces the group public key size to $O\left(N^{1 / 2}\right)$.

In anonymous credentials, Tsang et al. [51] showed how to blacklist users without compromising their anonymity or involving a trusted third party. Their schemes either rely on accumulators (which may be problematic in our setting) or have linear proving complexity in the number of revocations. Camenisch, Kohlweiss and Soriente [25] dealt with revocations in anonymous credentials by periodically updating users credentials in which a specific attribute indicates a validity period. In group signatures, their technique would place an important

\footnotetext{
${ }^{2}$ In [14], signing keys consist of pairs $\left(g^{1 /(\omega+s)}, s\right) \in \mathbb{G} \times \mathbb{Z}_{p}$, where $\omega \in \mathbb{Z}_{p}$ is the secret key of the group manager, and the revocation method relies on the availability of the exponent $s \in \mathbb{Z}_{p}$. In the standard model, the Groth-Sahai techniques would require to turn the membership certificates into triples $\left(g^{1 /(\omega+s)}, g^{s}, u^{s}\right)$, for some $u \in \mathbb{G}$ (as in [19]), which is not compatible with the revocation mechanism.
} 
burden on the group manager who would have to generate updates for each unrevoked individual credential.

While, for various reasons, none of the above constructions conveniently supports large groups, a highly scalable revocation mechanism borrowed from the literature on broadcast encryption was recently described by Libert, Peters and Yung 44] (LPY). Using the Subset Cover framework of Naor, Naor and Lotspiech [47. (NNL), they described a history-independent revocable group signature in the standard model with constant verification time and at most polylogarithmic complexity in other parameters. The technique of [44] blends well with structurepreserving signatures [12] and the Groth-Sahai proofs [36]. The best tradeoff of [44] builds on the Subset Difference (SD) method [47] in its public-key variant due to Dodis and Fazio 31. It features constant signature size and verification time, $O(\log N)$-size group public keys, revocation lists of size $O(r)$ (as in standard PKIs and group signatures with verifier-local revocation) and membership certificates of size $O\left(\log ^{3} N\right)$. This can be reduced to $O(\log N)$ using the Complete Subtree method [4] but revocation lists are then inflated by a factor of $O(\log N / r)$. Although the Layered Subset Difference method [37] allows for noticeable improvements, the constructions of 44 suffer from relatively large membership certificates. However, some logarithmic dependency on the group size is expected when basing revocation on a tree-like NNL methodology.

\subsection{Our Contributions}

To date, in the only scalable revocable group signatures with constant verification time in the standard model [4], group members have to store a polylogarithmic number of group elements. In many applications, however, this can rapidly become unwieldy even for moderately large groups: for example, using the Subset Difference method with $N=1000 \approx 2^{10}$, users may have to privately store thousands of group elements. In order to be competitive with other group signatures in the standard model such as [35] and still be able to revoke members while keeping them "stateless", it is highly desirable to reduce this complexity.

In this paper, we start with the approach of [44] so as to instantiate the Subset Difference method, but obtain private keys of constant size without degrading other performance criteria. This may sound somewhat surprising since, in the SD method, (poly)logarithmic complexities inherently seem inevitable in several metrics. Indeed, in the context of broadcast encryption [47, it requires private keys of size $O\left(\log ^{2} N\right)$ (and even $O\left(\log ^{3} N\right)$ in the public key setting [31] if the result of Boneh-Boyen-Goh [13] is used). Here, we reduce this overhead to a constant while the only dependency on $N$ is a $O(\log N)$-size group public key.

The key idea is as follows. As in the NNL framework, group members are assigned to a leaf of a binary tree and each unrevoked member should belong to exactly one subset in the cover of authorized leafs determined by the group manager. Instead of relying on hierarchical identity-based encryption [15 38 33. as in the public-key variant [31] of NNL, we use a novel way for users to noninteractively prove their membership of some generic subset of the SD method using a proof of constant size. 
To construct these "compact anonymous membership proofs", we use concise vector commitment schemes 4327, where each commitment can be opened w.r.t. individual coordinates in a space-efficient manner (namely, the size of a coordinate-wise opening does not depend on the length of the vector). These vector commitments interact nicely with the specific shape of subsets - as differences between two subtrees - in the SD method. Using them, we compactly encode as a vector the path from the user's leaf to the root. To provide evidence of their inclusion in one of the SD subsets, group members successively prove the equality and the inequality between two coordinates of their vector (i.e., two nodes of the path from their leaf to the root) and specific node labels indicated by an appropriate entry of the revocation list. This is where the position-wise openability of concise commitments is very handy. Of course, for anonymity purposes, the relevant entry of the revocation list only appears in committed form in the group signature. In order to prove that he is using a legal entry of the revocation list, the user generates a set membership proof [23] and proves knowledge of a signature from the group manager on the committed RL entry.

Our technique allows making the most of the LPY approach 44] by reducing the size of membership certificates to a small constant: at the cost of lengthening signatures by a factor of only 1.5, we obtain membership certificates consisting of only 9 group elements and a small integer. For $N=1000$, users' private keys are thus compressed by a multiplicative factor of several hundreds and this can only become more dramatic for larger groups. At the same time, our main scheme retains all the useful properties of 44]: like the construction of Nakanishi et al. [45, it does not require users to update their membership certificates at any time but, unlike [45], our group public key size is $O(\log N)$. Like the SD-based construction of 44, our system uses revocation lists of size $O(r)$, which is on par with Certificate Revocation Lists (CRLs) in PKIs. It is worth noting that RLs are not part of the group public key: verifiers only need to know the number of the latest revocation epoch and should not bother to read RLs entirely.

Eventually, our novel approach yields revocable group signatures that become competitive with the regular CRL approach in PKIs: signature generation and verification have constant cost, signatures and membership certificates being of $O(1)$-size while revocation lists have size $O(r)$. A detailed efficiency comparison with previous approaches is given in the full version of the paper. Finally, it is conceivable that our improved revocation technique can find applications beyond group signatures.

\section{Background}

\subsection{Bilinear Maps and Complexity Assumptions}

We use bilinear maps $e: \mathbb{G} \times \mathbb{G} \rightarrow \mathbb{G}_{T}$ over groups of prime order $p$ where $e(g, h) \neq 1_{\mathbb{G}_{T}}$ if and only if $g, h \neq 1_{\mathbb{G}}$. In these groups, we rely on hardness assumptions that are all non-interactive.

Definition 1 ([14]). The Decision Linear Problem (DLIN) in $\mathbb{G}$, is to distinguish the distributions $\left(g^{a}, g^{b}, g^{a c}, g^{b d}, g^{c+d}\right)$ and $\left(g^{a}, g^{b}, g^{a c}, g^{b d}, g^{z}\right)$, with 
$a, b, c, d \stackrel{R}{\leftarrow} \mathbb{Z}_{p}^{*}, z \stackrel{R}{\leftarrow} \mathbb{Z}_{p}^{*}$. The Decision Linear Assumption is the intractability of DLIN for any PPT distinguisher D.

Definition 2 ([12]). The $q$-Strong Diffie-Hellman problem $(q-S D H)$ in $\mathbb{G}$ is, given $\left(g, g^{a}, \ldots, g^{\left(a^{q}\right)}\right)$, for some $g \stackrel{R}{\leftarrow} \mathbb{G}$ and $a \stackrel{R}{\leftarrow} \mathbb{Z}_{p}$, to find a pair $\left(g^{1 /(a+s)}, s\right) \in \mathbb{G} \times \mathbb{Z}_{p}$.

We use a signature scheme proposed by Abe et al. [1], the security of which relies on this assumption.

Definition 3 ([1]). In a group $\mathbb{G}$, the $q$-Simultaneous Flexible Pairing Problem (q-SFP) is, given $\left(g_{z}, h_{z}, g_{r}, h_{r}, a, \tilde{a}, b, \tilde{b} \in \mathbb{G}\right)$ and $q \in \operatorname{poly}(\lambda)$ tuples $\left(z_{j}, r_{j}, s_{j}, t_{j}, u_{j}, v_{j}, w_{j}\right) \in \mathbb{G}^{7}$ such that

$$
\begin{gathered}
e(a, \tilde{a})=e\left(g_{z}, z_{j}\right) \cdot e\left(g_{r}, r_{j}\right) \cdot e\left(s_{j}, t_{j}\right), \\
e(b, \tilde{b})=e\left(h_{z}, z_{j}\right) \cdot e\left(h_{r}, u_{j}\right) \cdot e\left(v_{j}, w_{j}\right),
\end{gathered}
$$

to find a new tuple $\left(z^{\star}, r^{\star}, s^{\star}, t^{\star}, u^{\star}, v^{\star}, w^{\star}\right) \in \mathbb{G}^{7}$ satisfying relations (1) and such that $z^{\star} \notin\left\{1_{\mathbb{G}}, z_{1}, \ldots, z_{q}\right\}$.

The paper will appeal to an assumption that was implicitly introduced in [16].

Definition 4 ([16]). Let $\mathbb{G}$ be a group of prime order $p$. The $\ell$-Diffie-Hellman Exponent $(\ell-D H E)$ problem is, given elements $\left(g, g_{1}, \ldots, g_{\ell}, g_{\ell+2}, \ldots, g_{2 \ell}\right) \in$ $\mathbb{G}^{2 \ell}$ such that $g_{i}=g^{\left(\alpha^{i}\right)}$ for each $i$ and where $\alpha \stackrel{R}{\leftarrow} \mathbb{Z}_{p}^{*}$, to compute the missing element $g_{\ell+1}=g^{\left(\alpha^{\ell+1}\right)}$.

We actually need a stronger variant, used in [39], of the $\ell$-DHE assumption.

Definition 5. In a group $\mathbb{G}$ of prime order $p$, the Flexible $\ell$-Diffie-Hellman Exponent ( $\ell$-FlexDHE) problem is, given $\left(g, g_{1}, \ldots, g_{\ell}, g_{\ell+2}, \ldots, g_{2 \ell}\right) \in \mathbb{G}^{2 \ell}$ such that $g_{i}=g^{\left(\alpha^{i}\right)}$ for each $i$ and where $\alpha \stackrel{R}{\leftarrow} \mathbb{Z}_{p}^{*}$, to compute a non-trivial triple $\left(g^{\mu}, g_{\ell+1}^{\mu}, g_{2 \ell}^{\mu}\right) \in\left(\mathbb{G} \backslash\left\{1_{\mathbb{G}}\right\}\right)^{3}$, for some $\mu \in \mathbb{Z}_{p}^{*}$ and where $g_{\ell+1}=g^{\left(\alpha^{\ell+1}\right)}$.

The reason why we need to rely on the above assumption instead of the weaker $\ell$-DHE assumption is that, in our proofs, the exponent $\mu \in \mathbb{Z}_{p}$ will appear inside Groth-Sahai commitments [36], from which only values of the form $\left(g^{\mu}, g_{\ell+1}^{\mu}\right)$ will be efficiently extractable. The additional element $g_{2 \ell}^{\mu}$ will thus prevent the adversary from simply choosing $\mu=\alpha$ or $\mu=\alpha^{-1}$.

A proof of the generic hardness of the $\ell$-FlexDHE problem is given in 39 . We note that, while the strength of the assumption grows with $\ell, \ell$ is only logarithmic in the maximal number of users here.

\subsection{Groth-Sahai Proof Systems}

The fundamental Groth-Sahai (GS) techniques [36] can be based on the DLIN assumption, where they use prime order groups and a common reference string containing three vectors $\overrightarrow{f_{1}}, \overrightarrow{f_{2}}, \overrightarrow{f_{3}} \in \mathbb{G}^{3}$, where $\overrightarrow{f_{1}}=\left(f_{1}, 1, g\right), \overrightarrow{f_{2}}=\left(1, f_{2}, g\right)$ for 
some $f_{1}, f_{2} \in \mathbb{G}$. To commit to $X \in \mathbb{G}$, one chooses $r, s, t \stackrel{R}{\leftarrow} \mathbb{Z}_{p}^{*}$ and computes $\vec{C}=(1,1, X) \cdot{\overrightarrow{f_{1}}}^{r} \cdot{\overrightarrow{f_{2}}}^{s} \cdot{\overrightarrow{f_{3}}}^{t}$. In the soundness setting, we have $\vec{f}_{3}={\overrightarrow{f_{1}}}^{\xi_{1}} \cdot \vec{f}_{2} \xi_{2}$ where $\xi_{1}, \xi_{2} \in \mathbb{Z}_{p}^{*}$. Commitments $\vec{C}=\left(f_{1}^{r+\xi_{1} t}, f_{2}^{s+\xi_{2} t}, X \cdot g^{r+s+t\left(\xi_{1}+\xi_{2}\right)}\right)$ are then extractable using $\beta_{1}=\log _{g}\left(f_{1}\right), \beta_{2}=\log _{g}\left(f_{2}\right)$. In the witness indistinguishability (WI) setting, vectors $\overrightarrow{f_{1}}, \overrightarrow{f_{2}}, \overrightarrow{f_{3}}$ are linearly independent and $\vec{C}$ is a perfectly hiding commitment. Under the DLIN assumption, the two kinds of CRS are indistinguishable.

To commit to an exponent $x \in \mathbb{Z}_{p}$, one computes $\vec{C}=\vec{\varphi}^{x} \cdot{\overrightarrow{f_{1}}}^{r} \cdot{\overrightarrow{f_{2}}}^{s}$, where $r, s \stackrel{R}{\leftarrow} \mathbb{Z}_{p}^{*}$, using a CRS consisting of vectors $\vec{\varphi}, \overrightarrow{f_{1}}, \overrightarrow{f_{2}}$. In the perfect soundness setting, $\vec{\varphi}, \overrightarrow{f_{1}}, \overrightarrow{f_{2}}$ are linearly independent whereas, in the WI setting, choosing $\vec{\varphi}=\vec{f}_{1}^{\xi_{1}} \cdot \vec{f}_{2}{ }^{\xi_{2}}$ gives a perfectly hiding commitment.

To prove that committed variables satisfy a set of relations, the prover computes one commitment per variable and one proof element per relation. Such non-interactive witness indistinguishable (NIWI) proofs are available for pairingproduct equations, which are relations of the type

$$
\prod_{i=1}^{n} e\left(\mathcal{A}_{i}, \mathcal{X}_{i}\right) \cdot \prod_{i=1}^{n} \cdot \prod_{j=1}^{n} e\left(\mathcal{X}_{i}, \mathcal{X}_{j}\right)^{a_{i j}}=t_{T}
$$

for variables $\mathcal{X}_{1}, \ldots, \mathcal{X}_{n} \in \mathbb{G}$ and constants $t_{T} \in \mathbb{G}_{T}, \mathcal{A}_{1}, \ldots, \mathcal{A}_{n} \in \mathbb{G}, a_{i j} \in \mathbb{Z}_{p}$, for $i, j \in\{1, \ldots, n\}$. Efficient NIWI proofs also exist for multi-exponentiation equations, which are of the form

$$
\prod_{i=1}^{m} \mathcal{A}_{i}^{y_{i}} \cdot \prod_{j=1}^{n} \mathcal{X}_{j}^{b_{j}} \cdot \prod_{i=1}^{m} \cdot \prod_{j=1}^{n} \mathcal{X}_{j}^{y_{i} \gamma_{i j}}=T
$$

for variables $\mathcal{X}_{1}, \ldots, \mathcal{X}_{n} \in \mathbb{G}, y_{1}, \ldots, y_{m} \in \mathbb{Z}_{p}$ and constants $T, \mathcal{A}_{1}, \ldots, \mathcal{A}_{m} \in \mathbb{G}$, $b_{1}, \ldots, b_{n} \in \mathbb{Z}_{p}$ and $\gamma_{i j} \in \mathbb{G}$, for $i \in\{1, \ldots, m\}, j \in\{1, \ldots, n\}$.

In pairing-product equations, proofs for quadratic equations consist of 9 group elements whereas linear equations (i.e., where $a_{i j}=0$ for all $i, j$ in equation (2)) only demand 3 group elements each. Linear multi-exponentiation equations of the type $\prod_{i=1}^{m} \mathcal{A}_{i}^{y_{i}}=T$ demand 2 group elements.

Multi-exponentiation equations admit zero-knowledge (NIZK) proofs at no additional cost. On a simulated CRS (prepared for the WI setting), a trapdoor allows simulating proofs without using the witnesses.

\subsection{Structure-Preserving Signatures}

Many anonymity-related protocols (e.g., 281|2323]) require to sign elements of bilinear groups while maintaining the feasibility of conveniently proving that a committed signature is valid for a committed message.

Abe, Haralambiev and Ohkubo [12 (AHO) showed how to sign $n$ group elements using signatures consisting of $O(1)$ group elements. In the context of symmetric pairings, the description hereafter assumes public parameters 
$\mathrm{pp}=\left(\left(\mathbb{G}, \mathbb{G}_{T}\right), g\right)$ consisting of groups $\left(\mathbb{G}, \mathbb{G}_{T}\right)$ of order $p>2^{\lambda}$, where $\lambda \in \mathbb{N}$ is a security parameter, with a bilinear map $e: \mathbb{G} \times \mathbb{G} \rightarrow \mathbb{G}_{T}$ and a generator $g \in \mathbb{G}$.

Keygen(pp, $n)$ : given an upper bound $n \in \mathbb{N}$ on the number of group elements per signed message, choose generators $G_{r}, H_{r} \stackrel{R}{\leftarrow} \mathbb{G}$. Pick $\gamma_{z}, \delta_{z} \stackrel{R}{\leftarrow} \mathbb{Z}_{p}$ and $\gamma_{i}, \delta_{i} \stackrel{R}{\leftarrow} \mathbb{Z}_{p}$, for $i=1$ to $n$. Then, compute $G_{z}=G_{r}^{\gamma_{z}}, H_{z}=H_{r}^{\delta_{z}}$ and $G_{i}=G_{r}^{\gamma_{i}}, H_{i}=H_{r}^{\delta_{i}}$ for each $i \in\{1, \ldots, n\}$. Finally, choose $\alpha_{a}, \alpha_{b} \stackrel{R}{\leftarrow} \mathbb{Z}_{p}$ and define $A=e\left(G_{r}, g^{\alpha_{a}}\right)$ and $B=e\left(H_{r}, g^{\alpha_{b}}\right)$. The public key is defined to be

$$
p k=\left(G_{r}, H_{r}, G_{z}, H_{z},\left\{G_{i}, H_{i}\right\}_{i=1}^{n}, A, B\right) \in \mathbb{G}^{2 n+4} \times \mathbb{G}_{T}^{2}
$$

while the private key is $s k=\left(\alpha_{a}, \alpha_{b}, \gamma_{z}, \delta_{z},\left\{\gamma_{i}, \delta_{i}\right\}_{i=1}^{n}\right)$.

$\operatorname{Sign}\left(s k,\left(M_{1}, \ldots, M_{n}\right)\right)$ : to sign a vector $\left(M_{1}, \ldots, M_{n}\right) \in \mathbb{G}^{n}$ using the private key $s k=\left(\alpha_{a}, \alpha_{b}, \gamma_{z}, \delta_{z},\left\{\gamma_{i}, \delta_{i}\right\}_{i=1}^{n}\right)$, choose $\zeta, \rho_{a}, \rho_{b}, \omega_{a}, \omega_{b} \stackrel{R}{\leftarrow} \mathbb{Z}_{p}$ and compute $\theta_{1}=g^{\zeta}$ as well as

$$
\begin{array}{lll}
\theta_{2}=g^{\rho_{a}-\gamma_{z} \zeta} \cdot \prod_{i=1}^{n} M_{i}^{-\gamma_{i}}, & \theta_{3}=G_{r}^{\omega_{a}}, & \theta_{4}=g^{\left(\alpha_{a}-\rho_{a}\right) / \omega_{a}}, \\
\theta_{5}=g^{\rho_{b}-\delta_{z} \zeta} \cdot \prod_{i=1}^{n} M_{i}^{-\delta_{i}}, & \theta_{6}=H_{r}^{\omega_{b}}, & \theta_{7}=g^{\left(\alpha_{b}-\rho_{b}\right) / \omega_{b}}
\end{array}
$$

The signature consists of $\sigma=\left(\theta_{1}, \theta_{2}, \theta_{3}, \theta_{4}, \theta_{5}, \theta_{6}, \theta_{7}\right)$.

$\operatorname{Verify}\left(p k, \sigma,\left(M_{1}, \ldots, M_{n}\right)\right)$ : parse $\sigma$ as $\left(\theta_{1}, \theta_{2}, \theta_{3}, \theta_{4}, \theta_{5}, \theta_{6}, \theta_{7}\right) \in \mathbb{G}^{7}$ and return 1 iff these equalities hold:

$$
\begin{aligned}
& A=e\left(G_{z}, \theta_{1}\right) \cdot e\left(G_{r}, \theta_{2}\right) \cdot e\left(\theta_{3}, \theta_{4}\right) \cdot \prod_{i=1}^{n} e\left(G_{i}, M_{i}\right), \\
& B=e\left(H_{z}, \theta_{1}\right) \cdot e\left(H_{r}, \theta_{5}\right) \cdot e\left(\theta_{6}, \theta_{7}\right) \cdot \prod_{i=1}^{n} e\left(H_{i}, M_{i}\right) .
\end{aligned}
$$

The scheme was proved [12] existentially unforgeable under chosen-message attacks under the $q$-SFP assumption, where $q$ is the number of signing queries.

Signatures can be publicly re-randomized to obtain a different signature $\left\{\theta_{i}^{\prime}\right\}_{i=1}^{7} \leftarrow \operatorname{ReR} \operatorname{Rand}(p k, \sigma)$ on the same message $\left(M_{1}, \ldots, M_{n}\right)$. After randomization, we have $\theta_{1}^{\prime}=\theta_{1}$ while $\left\{\theta_{i}^{\prime}\right\}_{i=2}^{7}$ are uniformly distributed among the values such that $e\left(G_{r}, \theta_{2}^{\prime}\right) \cdot e\left(\theta_{3}^{\prime}, \theta_{4}^{\prime}\right)=e\left(G_{r}, \theta_{2}\right) \cdot e\left(\theta_{3}, \theta_{4}\right)$ and $e\left(H_{r}, \theta_{5}^{\prime}\right) \cdot e\left(\theta_{6}^{\prime}, \theta_{7}^{\prime}\right)=$ $e\left(H_{r}, \theta_{5}\right) \cdot e\left(\theta_{6}, \theta_{7}\right)$. Moreover, $\left\{\theta_{i}^{\prime}\right\}_{i \in\{3,4,6,7\}}$ are statistically independent of the message and other signature components. This implies that, in privacy-preserving protocols, re-randomized $\left\{\theta_{i}^{\prime}\right\}_{i \in\{3,4,6,7\}}$ can be safely given in the clear as long as $\left(M_{1}, \ldots, M_{n}\right)$ and $\left\{\theta_{i}^{\prime}\right\}_{i \in\{1,2,5\}}$ are given in committed form.

\subsection{Vector Commitment Schemes}

We use concise vector commitment schemes, where commitments can be opened with a short de-commitment string for each individual coordinate. Such commitments based on ideas from [16 24 were described by Libert and Yung [43] 
and, under weaker assumptions, by Catalano and Fiore [27. In [43, the commitment key is $c k=\left(g, g_{1}, \ldots, g_{\ell}, g_{\ell+2}, \ldots, g_{2 \ell}\right) \in \mathbb{G}^{2 \ell}$, where $g_{i}=g^{\left(\alpha^{i}\right)}$ for each $i$. The trapdoor of the commitment is $g_{\ell+1}$, which does not appear in $c k$. To commit to a vector $\vec{m}=\left(m_{1}, \ldots, m_{\ell}\right)$, the committer picks $r \stackrel{R}{\leftarrow} \mathbb{Z}_{p}$ and computes $C=g^{r} \cdot \prod_{\kappa=1}^{\ell} g_{\ell+1-\kappa}^{m_{\kappa}}$. A single group element $W_{i}=g_{i}^{r} \cdot \prod_{\kappa=1, \kappa \neq i}^{\ell} g_{\ell+1-\kappa+i}^{m_{\kappa}}$ provides evidence that $m_{i}$ is the $i$-th component of $\vec{m}$ as it satisfies the relation $e\left(g_{i}, C\right)=e\left(g, W_{i}\right) \cdot e\left(g_{1}, g_{\ell}\right)^{m_{i}}$. The infeasibility of opening a commitment to two distinct messages for some coordinate $i$ relies on the $\ell$-DHE assumption. For our purposes, we only rely on the position-wise binding property of vector commitments and do not need them to be hiding. The randomizer $r$ will thus be removed from the expression of $C$.

\subsection{The NNL Framework for Broadcast Encryption}

The important Subset Cover framework [4] considers secret-key broadcast encryption schemes with $N=2^{\ell}$ registered receivers. Each receiver is associated with a leaf of a complete binary tree $\mathrm{T}$ of height $\ell$ where each node is assigned a secret key. If $\mathcal{N}$ denotes the universe of users and $\mathcal{R} \subset \mathcal{N}$ is the set of revoked receivers, the framework's idea is to partition the set of non-revoked users into $m$ disjoint subsets $S_{1}, \ldots, S_{m}$ such that $\mathcal{N} \backslash \mathcal{R}=S_{1} \cup \ldots \cup S_{m}$. Depending on the way to divide $\mathcal{N} \backslash \mathcal{R}$, different tradeoffs are possible.

The Subset Difference (SD) method yields a transmission cost of $O(|\mathcal{R}|)$ and a storage complexity in $O\left(\log ^{2} N\right)$. For each node $x_{j} \in \mathrm{T}$, we call $\mathrm{T}_{x_{j}}$ the subtree rooted at $x_{j}$. The unrevoked set $\mathcal{N} \backslash \mathcal{R}$ is partitioned into disjoint subsets $S_{k_{1}, u_{1}}, \ldots, S_{k_{m}, u_{m}}$. For each $i \in\{1, \ldots, m\}$, the subset $S_{k_{i}, u_{i}}$ is determined by a node $x_{k_{i}}$ and one of its descendants $x_{u_{i}}$ - which are called primary and secondary roots of $S_{k_{i}, u_{i}}$, respectively - and it consists of the leaves of $\mathrm{T}_{x_{k_{i}}}$ that are not in $\mathrm{T}_{x_{u_{i}}}$. Each user belongs to many generic subsets, so that the number of subsets bounded by $m=2 \cdot|\mathcal{R}|-1$, as proved in [47].

In the broadcast encryption scenario, a sophisticated key distribution process is necessary to avoid a prohibitive storage overhead. Each subset $S_{k_{i}, u_{i}}$ is assigned a "proto-key" $P_{x_{k_{i}}, x_{u_{i}}}$ that allows deriving the actual symmetric encryption key $K_{k_{i}, u_{i}}$ for $S_{k_{i}, u_{i}}$ and as well as proto-keys $P_{x_{k_{i}}, x_{u_{l}}}$ for any descendant $x_{u_{l}}$ of $x_{u_{i}}$. Eventually, each user has to store $O\left(\log ^{2} N\right)$ keys. In the setting of group signatures, we will show that, somewhat unexpectedly, the use of vector commitment schemes allows reducing the private storage to a constant: the size of users' private keys only depends on the security parameter $\lambda$, and not on $N$.

\subsection{Revocable Group Signatures}

As in 4544, we consider schemes that have their lifetime divided into revocation epochs at the beginning of which group managers update their revocation lists.

The syntax and the security model are similar to those used by Kiayias and Yung [40. Like the Bellare-Shi-Zhang model [10], the Kiayias-Yung model 
assumes an interactive join protocol whereby the user becomes a group member by interacting with the group manager.

Syntax. We denote by $N \in$ poly $(\lambda)$ the maximal number of group members. At the beginning of each revocation epoch $t$, the group manager publicizes an up-todate revocation list $R L_{t}$ and we denote by $\mathcal{R}_{t} \subset\{1, \ldots, N\}$ the corresponding set of revoked users (we assume that $\mathcal{R}_{t}$ is part of $R L_{t}$ ). A revocable group signature (R-GS) scheme consists of the following algorithms or protocols.

$\operatorname{Setup}(\lambda, N)$ : given a security parameter $\lambda \in \mathbb{N}$ and a maximal number of group members $N \in \mathbb{N}$, this algorithm (which is run by a trusted party) generates a group public key $\mathcal{Y}$, the group manager's private key $\mathcal{S}_{\mathrm{GM}}$ and the opening authority's private key $\mathcal{S}_{\mathrm{OA}} \cdot \mathcal{S}_{\mathrm{GM}}$ and $\mathcal{S}_{\mathrm{OA}}$ are given to the appropriate authority while $\mathcal{Y}$ is publicized. The algorithm initializes a public state $S t$ consisting of set and string data structures $S t_{\text {users }}=\emptyset$ and $S t_{\text {trans }}=\epsilon$.

Join: is an interactive protocol between the group manager GM and a prospective group member $\mathcal{U}_{i}$. The protocol involves two interactive Turing machines $J_{\text {user }}$ and $J_{G M}$ that both take as input $\mathcal{Y}$. The execution, denoted as $\left[\mathrm{J}_{\text {user }}(\lambda, \mathcal{Y}), \mathrm{J}_{\mathrm{GM}}\left(\lambda, S t, \mathcal{Y}, \mathcal{S}_{\mathrm{GM}}\right)\right]$, ends with $\mathcal{U}_{i}$ obtaining a membership secret $\mathrm{sec}_{i}$, that no one else knows, and a membership certificate cert ${ }_{i}$. If the protocol is successful, the group manager updates the public state $S t$ by setting $S t_{\text {users }}:=S t_{\text {users }} \cup\{i\}$ as well as $S t_{\text {trans }}:=S t_{\text {trans }} \|\left\langle i\right.$, transcript $\left._{i}\right\rangle$.

Revoke: is a (possibly probabilistic) algorithm allowing the GM to generate an updated revocation list $R L_{t}$ for the new revocation epoch $t$. It takes as input a public key $\mathcal{Y}$ and a set $\mathcal{R}_{t} \subset S t_{\text {users }}$ that identifies the users to be revoked. It outputs an updated revocation list $R L_{t}$ for epoch $t$.

Sign: given a revocation epoch $t$ with its revocation list $R L_{t}$, a membership certificate $\operatorname{cert}_{i}$, a membership secret $\sec _{i}$ and a message $M$, this algorithm outputs $\perp$ if $i \in \mathcal{R}_{t}$ and a signature $\sigma$ otherwise.

Verify: given a signature $\sigma$, a revocation epoch $t$, the corresponding revocation list $R L_{t}$, a message $M$ and a group public key $\mathcal{Y}$, this deterministic algorithm returns either 0 or 1 .

Open: takes as input a message $M$, a valid signature $\sigma$ w.r.t. $\mathcal{Y}$ for the indicated revocation epoch $t$, the opening authority's private key $\mathcal{S}_{\mathrm{OA}}$ and the public state $S t$. It outputs $i \in S t_{\text {users }} \cup\{\perp\}$, which is the identity of a group member or a symbol indicating an opening failure.

A R-GS scheme must satisfy three security notions that are formally defined in the full version of the paper. The first one is called security against misidentification attacks. It requires that, even if the adversary can introduce and revoke users at will, it cannot produce a signature that traces outside the set of unrevoked adversarially-controlled users. The notion of security against framing attacks captures that under no circumstances should an honest user be held accountable for messages that he did not sign, even if the whole system conspires against that user. Finally, the notion of anonymity is also defined (by granting the adversary access to a signature opening oracle) as in the models of [10 40]. 


\section{A Revocable Group Signature with Compact Keys and Constant Verification Time}

The number of users is assumed to be $N=2^{\ell-1} \in$ poly $(\lambda)$, for some integer $\ell$, so that each group member is assigned to a leaf of the tree. Each node is assigned a unique identifier. For simplicity, the root is identified by $\operatorname{ID}(\epsilon)=1$ and, for each other node $x$, we define the identifier $\operatorname{ID}(x) \in\{1, \ldots, 2 N-1\}$ to be $\operatorname{ID}(x)=2 \cdot \operatorname{ID}($ parent $(x))+b$, where parent $(x)$ denotes $x$ 's father in the tree and $b=0$ (resp. $b=1$ ) if $x$ is the left (resp. right) child of its father. The root of the tree is assigned the identifier $\mathrm{ID}_{\epsilon}=1$.

At the beginning of each revocation epoch $t$, the GM generates an up-to-date revocation list $R L_{t}$ containing one entry for each generic subset $S_{k_{1}, u_{1}}, \ldots, S_{k_{m}, u_{m}}$ produced by the Subset Difference method. These subsets are encoded in such a way that unrevoked users can anonymously prove their membership of one of them. Our technique allows to do this using a proof of constant size.

In the generation of $R L_{t}$, for each $i \in\{1, \ldots, m\}$, if $x_{k_{i}}$ (resp. $x_{u_{i}}$ ) denotes the primary (resp. secondary) root of $S_{k_{i}, u_{i}}$, the GM encodes $S_{k_{i}, u_{i}}$ as a vector of group elements $R_{i}$ that determines the levels of nodes $x_{k_{i}}$ and $x_{u_{i}}$ in the tree (which are called $\phi_{i}$ and $\psi_{i}$ hereafter) and the identifiers $\operatorname{ID}\left(x_{k_{i}}\right)$ and $\operatorname{ID}\left(x_{u_{i}}\right)$. Then, the vector $R_{i}$ is authenticated by means of a structure preserving signature $\Theta_{i}$, which is included in $R L_{t}$ so as to serve in a set membership proof [23].

During the join protocol, users obtain from the GM a structure-preserving signature on a compact encoding $C_{v}$ - which is computed as a commitment to a vector of node identifiers $\left(I_{1}, \ldots, I_{\ell}\right)$ - of the path $\left(I_{1}, \ldots, I_{\ell}\right)$ between their leaf $v$ and the root $\epsilon$. This path is encoded as a single group element.

In order to anonymously prove his non-revocation, a group member $\mathcal{U}_{i}$ uses $R L_{t}$ to determine the generic subset $S_{k_{l}, u_{l}}$, with $l \in\{1, \ldots, m\}$, where his leaf $v_{i}$ lies. He commits to the corresponding vector of group elements $R_{l}$ that encodes the node identifiers $\operatorname{ID}\left(x_{k_{l}}\right)$ and $\operatorname{ID}\left(x_{u_{l}}\right)$ of the primary and secondary roots of $S_{k_{l}, u_{l}}$ at levels $\phi_{l}$ and $\psi_{l}$, respectively. If $\left(I_{1}, \ldots, I_{\ell}\right)$ identifies the path from his leaf $v_{i}$ to $\epsilon$, the unrevoked member $\mathcal{U}_{i}$ generates a membership proof for the subset $S_{k_{l}, u_{l}}$ by proving that $\operatorname{ID}\left(x_{k_{l}}\right)=I_{\phi_{l}}$ and $\operatorname{ID}\left(x_{u_{l}}\right) \neq I_{\psi_{l}}$ (in other words, that $x_{k_{l}}$ is an ancestor of $v_{i}$ and $x_{u_{l}}$ is not). To succinctly prove these statements, $\mathcal{U}_{i}$ uses the properties of the commitment scheme recalled in Section 2.4. Finally, in order to convince the verifier that he used a legal element of $R L_{t}, \mathcal{U}_{i}$ follows the technique of [23] and proves knowledge of a signature $\Theta_{l}$ on the committed vector of group elements $R_{l}$. By doing so, $\mathcal{U}_{i}$ thus provides evidence that his leaf $v_{i}$ is a member of some authorized subset $S_{k_{l}, u_{l}}$ without revealing $l$.

In order to obtain the strongest flavor of anonymity (i.e., where the adversary has access to a signature opening oracle), the scheme uses Kiltz's tag-based encryption scheme [41] as in Groth's construction [35]. In non-frameability concerns, the group member $\mathcal{U}_{i}$ also generates a weak Boneh-Boyen signature [12] (which yields a fully secure signature when combined with a one-time signature) using $x=\log _{g}(X)$, where $X \in \mathbb{G}$ is a group element certified by the GM and bound to the path $\left(I_{1}, \ldots, I_{\ell}\right)$ during the join protocol. 


\subsection{Construction}

As in the security models of [1040, we assume that, before joining the group, user $\mathcal{U}_{i}$ chooses a long term key pair (usk $[i]$, upk $[i]$ ) and registers it in some PKI.

$\operatorname{Setup}(\lambda, N)$ : given a security parameter $\lambda \in \mathbb{N}$ and $N=2^{\ell-1}$,

1. Choose bilinear groups $\left(\mathbb{G}, \mathbb{G}_{T}\right)$ of prime order $p>2^{\lambda}$, with $g \stackrel{R}{\leftarrow} \mathbb{G}$.

2. Define $n_{0}=2$ and $n_{1}=5$. Generate two key pairs $\left(s k_{\mathrm{AHO}}^{(0)}, p k_{\mathrm{AHO}}^{(0)}\right)$ and $\left(s k_{\mathrm{AHO}}^{(1)}, p k_{\mathrm{AHO}}^{(1)}\right)$ for the AHO signature in order to sign messages of $n_{0}$ and $n_{1}$ group elements, respectively. These key pairs are

$$
\begin{aligned}
p k_{\mathrm{AHO}}^{(d)}=\left(G_{r}^{(d)}, H_{r}^{(d)}, G_{z}^{(d)}\right. & =G_{r}^{\gamma_{z}^{(d)}}, H_{z}^{(d)}=H_{r}^{\delta_{z}^{(d)}}, \\
\left\{G_{i}^{(d)}\right. & \left.\left.=G_{r}^{\gamma_{i}^{(d)}}, H_{i}^{(d)}=H_{r}^{\delta_{i}^{(d)}}\right\}_{i=1}^{n_{d}}, A^{(d)}, B^{(d)}\right)
\end{aligned}
$$

and $s k_{\mathrm{AHO}}^{(d)}=\left(\alpha_{a}^{(d)}, \alpha_{b}^{(d)}, \gamma_{z}^{(d)}, \delta_{z}^{(d)},\left\{\gamma_{i}^{(d)}, \delta_{i}^{(d)}\right\}_{i=1}^{n_{d}}\right)$, where $d \in\{0,1\}$.

3. Generate a public key $c k=\left(g_{1}, \ldots, g_{\ell}, g_{\ell+2}, \ldots, g_{2 \ell}\right) \in \mathbb{G}^{2 \ell-1}$ for vectors of dimension $\ell$ in the vector commitment scheme recalled in section 2.4 The trapdoor $g_{\ell+1}$ is not needed and can be discarded.

4. As a CRS for the NIWI proof system, select vectors $\mathbf{f}=\left(\overrightarrow{f_{1}}, \overrightarrow{f_{2}}, \overrightarrow{f_{3}}\right)$ s.t. $\overrightarrow{f_{1}}=\left(f_{1}, 1, g\right) \in \mathbb{G}^{3}, \overrightarrow{f_{2}}=\left(1, f_{2}, g\right) \in \mathbb{G}^{3}$, and $\overrightarrow{f_{3}}=\vec{f}_{1} \xi_{1} \cdot \vec{f}_{2} \xi_{2}$, with $f_{1}=g^{\beta_{1}}, f_{2}=g^{\beta_{2}} \stackrel{R}{\leftarrow} \mathbb{G}$ and $\beta_{1}, \beta_{2}, \xi_{1}, \xi_{2} \stackrel{R}{\leftarrow} \mathbb{Z}_{p}^{*}$. We also define the vector $\vec{\varphi}=\overrightarrow{f_{3}} \cdot(1,1, g)$.

5. Choose $(U, V) \stackrel{R}{\leftarrow} \mathbb{G}^{2}$ that, together with generators $f_{1}, f_{2}, g \in \mathbb{G}$, will form a public encryption key.

6. Select a strongly unforgeable one-time signature $\Sigma=(\mathcal{G}, \mathcal{S}, \mathcal{V})$.

7. Set $\mathcal{S}_{\mathrm{GM}}:=\left(s k_{\mathrm{AHO}}^{(0)}, s k_{\mathrm{AHO}}^{(1)}\right), \mathcal{S}_{\mathrm{OA}}:=\left(\beta_{1}, \beta_{2}\right)$ as authorities' private keys and the group public key is

$\mathcal{Y}:=\left(g, p k_{\mathrm{AHO}}^{(0)}, p k_{\mathrm{AHO}}^{(1)}, c k=\left(g_{1}, \ldots, g_{\ell}, g_{\ell+2}, \ldots, g_{2 \ell}\right), \mathbf{f}, \vec{\varphi},(U, V), \Sigma\right)$.

Join $\left(\mathrm{GM}, \mathcal{U}_{i}\right)$ : the GM and the prospective user $\mathcal{U}_{i}$ run the following protocol $\left[\mathrm{J}_{\text {user }}(\lambda, \mathcal{Y}), \mathrm{J}_{\mathrm{GM}}\left(\lambda, S t, \mathcal{Y}, \mathcal{S}_{\mathrm{GM}}\right)\right]$ :

1. $\mathrm{J}_{\text {user }}(\lambda, \mathcal{Y})$ draws $x \stackrel{R}{\leftarrow} \mathbb{Z}_{p}$ and sends $X=g^{x}$ to $\mathrm{J}_{\mathrm{GM}}\left(\lambda, S t, \mathcal{Y}, \mathcal{S}_{\mathrm{GM}}\right)$. If $X \in \mathbb{G}$ already appears in some entry transcript ${ }_{j}$ of the database $S t_{\text {trans }}$, $\mathrm{J}_{\mathrm{GM}}$ halts and returns $\perp$ to $J_{\text {user }}$.

2. $\mathrm{J}_{\mathrm{GM}}$ assigns to $\mathcal{U}_{i}$ an available leaf $v$ of identifier $\operatorname{ID}(v)$ in the tree $\mathrm{T}$. Let $x_{1}, \ldots, x_{\ell}$ be the path from $x_{\ell}=v$ to the root $x_{1}=\epsilon$ of $\mathrm{T}$. Let also $\left(I_{1}, \ldots, I_{\ell}\right)=\left(\operatorname{ID}\left(x_{1}\right), \ldots, \operatorname{ID}\left(x_{\ell}\right)\right)$ be the corresponding vector of identifiers (with $I_{1}=1$ and $I_{\ell}=\operatorname{ID}(v) \in\{N, \ldots, 2 N-1\}$ ). Then, $\mathrm{J}_{\mathrm{GM}}$ does the following.

a. Encode $\left(I_{1}, \ldots, I_{\ell}\right)$ as $C_{v}=\prod_{\kappa=1}^{\ell} g_{\ell+1-\kappa}^{I_{\kappa}}=g_{\ell}^{I_{1}} \cdots g_{1}^{I_{\ell}}$. 
b. Using $s k_{\text {AHO }}^{(0)}$, generate an AHO signature $\sigma_{v}=\left(\theta_{v, 1}, \ldots, \theta_{v, 7}\right)$ on the pair $\left(X, C_{v}\right) \in \mathbb{G}^{2}$ so as to bind the encoded path $C_{v}$ to the value $X$ that identifies $\mathcal{U}_{i}$.

3. $\mathrm{J}_{\mathrm{GM}}$ sends $\operatorname{ID}(v) \in\{N, \ldots, 2 N-1\}$ and $C_{v}$ to $\mathrm{J}_{\text {user }}$ that halts if $\operatorname{ID}(v) \notin$ $\{N, \ldots, 2 N-1\}$ or if $C_{v}$ is found incorrect. Otherwise, Juser sends a signature $\operatorname{sig}_{i}=\operatorname{Sign}_{\text {usk }[i]}\left(X \|\left(I_{1}, \ldots, I_{\ell}\right)\right)$ to $J_{\mathrm{GM}}$.

4. $J_{\mathrm{GM}}$ checks that $\operatorname{Verify} \mathrm{upk}_{\mathrm{u} i i}\left(\left(X \|\left(I_{1}, \ldots, I_{\ell}\right)\right), \operatorname{sig}_{i}\right)=1$. If not $\mathrm{J}_{\mathrm{GM}}$ aborts. Otherwise, $\mathrm{J}_{\mathrm{GM}}$ returns the AHO signature $\sigma_{v}$ to $\mathrm{J}_{\text {user }}$ and stores the transcript transcript ${ }_{i}=\left(X, \operatorname{ID}(v), C_{v}, \sigma_{v}\right.$, sig $\left._{i}\right)$ in the database $S t_{\text {trans }}$.

5. Juser defines cert ${ }_{i}=\left(\operatorname{ID}(v), X, C_{v}, \sigma_{v}\right) \in\{N, \ldots, 2 N-1\} \times \mathbb{G}^{9}$, where $X$ will identify $\mathcal{U}_{i}$. The membership secret $\sec _{i}$ is defined as $\sec _{i}=x \in \mathbb{Z}_{p}$.

$\operatorname{Revoke}\left(\mathcal{Y}, \mathcal{S}_{\mathrm{GM}}, t, \mathcal{R}_{t}\right)$ : Parse $\mathcal{S}_{\mathrm{GM}}$ as $\mathcal{S}_{\mathrm{GM}}:=\left(s k_{\mathrm{AHO}}^{(0)}, s k_{\mathrm{AHO}}^{(1)}\right)$.

1. Using the covering algorithm of the SD method, find a cover of the unrevoked user set $\{1, \ldots, N\} \backslash \mathcal{R}_{t}$ as the union of disjoint subsets of the form $S_{k_{1}, u_{1}}, \ldots, S_{k_{m}, u_{m}}$, with $m \leq 2 \cdot\left|\mathcal{R}_{t}\right|-1$.

2. For $i=1$ to $m$, do the following.

a. Consider $S_{k_{i}, u_{i}}$ as the difference between sub-trees rooted at an internal node $x_{k_{i}}$ and one of its descendants $x_{u_{i}}$. Let $\phi_{i}, \psi_{i} \in\{1, \ldots, \ell\}$ be the depths of $x_{k_{i}}$ and $x_{u_{i}}$, respectively, in $\mathrm{T}$ assuming that the root $\epsilon$ is at depth 1. Encode $S_{k_{i}, u_{i}}$ as a vector $\left(g_{\phi_{i}}, g_{1}^{\operatorname{ID}\left(x_{k_{i}}\right)}, g_{\psi_{i}}, g^{\operatorname{ID}\left(x_{u_{i}}\right)}\right)$.

b. To authenticate $S_{k_{i}, u_{i}}$ and bind it to the revocation epoch $t$, use $s k_{\text {AHO }}^{(1)}$ to generate an AHO signature $\Theta_{i}=\left(\Theta_{i, 1}, \ldots, \Theta_{i, 7}\right) \in \mathbb{G}^{7}$ on the message $R_{i}=\left(g^{t}, g_{\phi_{i}}, g_{1}^{\mathrm{ID}\left(x_{k_{i}}\right)}, g_{\psi_{i}}, g^{\mathrm{ID}\left(x_{u_{i}}\right)}\right) \in \mathbb{G}^{5}$, where the epoch number $t$ is interpreted as an element of $\mathbb{Z}_{p}$.

Return the revocation data

$$
R L_{t}=\left(t, \mathcal{R}_{t},\left\{\phi_{i}, \psi_{i}, \operatorname{ID}\left(x_{k_{i}}\right), \operatorname{ID}\left(x_{u_{i}}\right), \Theta_{i}=\left(\Theta_{i, 1}, \ldots, \Theta_{i, 7}\right)\right\}_{i=1}^{m}\right) .
$$

$\operatorname{Sign}\left(\mathcal{Y}, t, R L_{t}, \operatorname{cert}_{i}, \sec _{i}, M\right)$ : return $\perp$ if $i \in \mathcal{R}_{t}$. Otherwise, to $\operatorname{sign} M \in\{0,1\}^{*}$, generate a one-time signature key pair $(\mathrm{SK}, \mathrm{VK}) \leftarrow \mathcal{G}(\lambda)$. Parse cert ${ }_{i}$ as cert $_{i}=\left(\operatorname{ID}\left(v_{i}\right), X, C_{v_{i}}, \sigma_{v_{i}}\right) \in\{N, \ldots, 2 N-1\} \times \mathbb{G}^{9}$ and $\sec _{i}$ as $x \in \mathbb{Z}_{p}$. Let $\epsilon=x_{1}, \ldots, x_{\ell}=v_{i}$ be the path connecting $v_{i}$ to the root $\epsilon$ of $\mathrm{T}$ and let $\left(I_{1}, \ldots, I_{\ell}\right)=\left(\operatorname{ID}\left(x_{1}\right), \ldots, \operatorname{ID}\left(x_{\ell}\right)\right)$ be the vector of node identifiers. First, $\mathcal{U}_{i}$ generates a commitment $\operatorname{com}_{C_{v_{i}}}$ to the encoding $C_{v_{i}}$ of the path $\left(I_{1}, \ldots, I_{\ell}\right)$ from $v_{i}$ to the root. Then, he does the following.

1. Using $R L_{t}$, find the set $S_{k_{l}, u_{l}}$, with $l \in\{1, \ldots, m\}$, containing the leaf $v_{i}$ identified by $\operatorname{ID}\left(v_{i}\right)$. Let $x_{k_{l}}$ and $x_{u_{l}}$ denote the primary and secondary roots of $S_{k_{l}, u_{l}}$ at depths $\phi_{l}$ and $\psi_{l}$, respectively. Since $x_{k_{l}}$ is an ancestor of $v_{i}$ but $x_{u_{l}}$ is not, it must be the case that $I_{\phi_{l}}=\operatorname{ID}\left(x_{k_{l}}\right)$ and $I_{\psi_{l}} \neq \operatorname{ID}\left(x_{u_{l}}\right)$.

2. To prove that $v_{i}$ belongs to $S_{k_{l}, u_{l}}$ without leaking $l, \mathcal{U}_{i}$ first re-randomizes the $l$-th AHO signature $\Theta_{l}$ of $R L_{t}$ as $\left\{\Theta_{l, i}^{\prime}\right\}_{i=1}^{7} \leftarrow \operatorname{Re} \operatorname{Rand}\left(p k_{\text {AHO }}^{(1)}, \Theta_{l}\right)$. 
Then, he commits to the $l$-th revocation message

$$
R_{l}=\left(R_{l, 1}, R_{l, 2}, R_{l, 3}, R_{l, 4}, R_{l, 5}\right)=\left(g^{t}, g_{\phi_{l}}, g_{1}^{\operatorname{ID}\left(x_{k_{l}}\right)}, g_{\psi_{l}}, g^{\operatorname{ID}\left(x_{u_{l}}\right)}\right)
$$

and its signature $\Theta_{l}^{\prime}=\left(\Theta_{l, 1}^{\prime}, \ldots, \Theta_{l, 7}^{\prime}\right)$ by computing Groth-Sahai commitments $\left\{\operatorname{com}_{R_{l, \tau}}\right\}_{\tau=2}^{5},\left\{\operatorname{com}_{\Theta_{l, j}^{\prime}}\right\}_{j \in\{1,2,5\}}$ to $\left\{R_{l, \tau}\right\}_{\tau=2}^{5}$ and $\left\{\Theta_{l, j}^{\prime}\right\}_{j \in\{1,2,5\}}$.

a. To prove that $I_{\phi_{l}}=\operatorname{ID}\left(x_{k_{l}}\right), \mathcal{U}_{i}$ computes $W_{\phi_{l}}=\prod_{\kappa=1, \kappa \neq \phi_{l}}^{\ell} g_{\ell+1-\kappa+\phi_{l}}^{I_{\kappa}}$

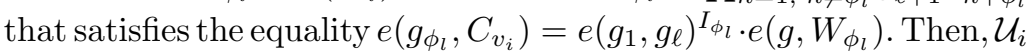
generates a commitment $\operatorname{com}_{W_{\phi_{l}}}$ to $W_{\phi_{l}}$. He computes a NIWI proof $\pi_{e q}$ that committed variables $\left(R_{l, 2}, R_{l, 3}, C_{v_{i}}, W_{\phi_{l}}\right)$ satisfy

$$
e\left(R_{l, 2}, C_{v_{i}}\right)=e\left(R_{l, 3}, g_{\ell}\right) \cdot e\left(g, W_{\phi_{l}}\right) .
$$

b. To prove that $I_{\psi_{l}} \neq \mathrm{ID}\left(x_{u_{l}}\right), \mathcal{U}_{i}$ computes $W_{\psi_{l}}=\prod_{\kappa=1, \kappa \neq \psi_{l}}^{\ell} g_{\ell+1-\kappa+\psi_{l}}^{I_{\kappa}}$ that satisfies the equality $e\left(g_{\psi_{l}}, C_{v_{i}}\right)=e\left(g_{1}, g_{\ell}\right)^{I_{\psi_{l}}} \cdot e\left(g, W_{\psi_{l}}\right)$. Then, he computes a commitment $\operatorname{com}_{W_{\psi_{l}}}$ to $W_{\psi_{l}}$ as well as commitments $\operatorname{com}_{\Gamma_{l}}$ and $\left\{\operatorname{com}_{\Psi_{l, \tau}}\right\}_{\tau \in\{0,1,2 \ell\}}$ to the group elements

$$
\left(\Gamma_{l}, \Psi_{l, 0}, \Psi_{l, 1}, \Psi_{l, 2 \ell}\right)=\left(g^{1 /\left(I_{\psi_{l}}-\mathrm{ID}\left(x_{u_{l}}\right)\right)}, g^{I_{\psi_{l}}}, g_{1}^{I_{\psi_{l}}}, g_{2 \ell}^{I_{\psi_{l}}}\right) .
$$

Then, $\mathcal{U}_{i}$ proves that $\left(R_{l, 4}, R_{l, 5}, C_{v_{i}}, \Gamma_{l}, \Psi_{l, 0}, \Psi_{l, 1}, \Psi_{l, 2 \ell}\right)$ satisfy

$$
\begin{aligned}
e\left(R_{l, 4}, C_{v_{i}}\right) & =e\left(\Psi_{l, 1}, g_{\ell}\right) \cdot e\left(g, W_{\psi_{l}}\right), & e\left(\Psi_{l, 0} / R_{l, 5}, \Gamma_{l}\right)=e(g, g) \\
e\left(\Psi_{l, 1}, g\right) & =e\left(g_{1}, \Psi_{l, 0}\right), & e\left(\Psi_{l, 2 \ell}, g\right)=e\left(g_{2 \ell}, \Psi_{l, 0}\right) .
\end{aligned}
$$

We denote this NIWI proof by $\pi_{n e q}=\left(\pi_{n e q, 1}, \pi_{n e q, 2}, \pi_{n e q, 3}, \pi_{n e q, 4}\right)$.

3. $\mathcal{U}_{i}$ proves that the tuple $R_{l}$ of $(5)$ is a certified revocation message for epoch $t$ : namely, he computes a NIWI proof $\pi_{R_{l}}$ that committed message elements $\left\{R_{l, \tau}\right\}_{\tau=2}^{5}$ and signature components $\left\{\Theta_{l, j}^{\prime}\right\}_{j \in\{1,2,5\}}$ satisfy

$$
\begin{aligned}
A^{(1)} \cdot e\left(\Theta_{l, 3}^{\prime}, \Theta_{l, 4}^{\prime}\right)^{-1} \cdot e\left(G_{1}^{(1)}, g^{t}\right)^{-1}= & e\left(G_{z}^{(1)}, \Theta_{l, 1}^{\prime}\right) \cdot \\
& e\left(G_{r}^{(1)}, \Theta_{l, 2}^{\prime}\right) \cdot \prod_{\tau=2}^{5} e\left(G_{\tau}^{(1)}, R_{l, \tau}\right), \\
B^{(1)} \cdot e\left(\Theta_{l, 6}^{\prime}, \Theta_{l, 7}^{\prime}\right)^{-1} \cdot e\left(H_{1}^{(1)}, g^{t}\right)^{-1}= & e\left(H_{z}^{(1)}, \Theta_{l, 1}^{\prime}\right) \\
& \cdot e\left(H_{r}^{(1)}, \Theta_{l, 5}^{\prime}\right) \cdot \prod_{\tau=2}^{5} e\left(H_{\tau}^{(1)}, R_{l, \tau}\right),
\end{aligned}
$$

Since $\left\{\Theta_{l, j}^{\prime}\right\}_{j \in\{3,4,6,7\}}$ are constants, equations (9) are both linear and thus require 3 elements each. Hence, $\pi_{R_{l}}$ takes 6 elements altogether.

4. Let $\sigma_{v_{i}}=\left(\theta_{v_{i}, 1}, \ldots, \theta_{v_{i}, 7}\right)$ be the AHO signature on $\left(X, C_{v_{i}}\right)$. Compute a commitment $\operatorname{com}_{X}$ to $X$. Set $\left\{\theta_{v_{i}, j}^{\prime}\right\}_{j=1}^{7} \leftarrow \operatorname{ReRand}\left(p k_{\mathrm{AHO}}^{(0)}, \sigma_{v_{i}}\right)$ and generate commitments $\left\{\operatorname{com}_{\theta_{v_{i}, j}^{\prime}}\right\}_{j \in\{1,2,5\}}$ to $\left\{\theta_{v_{i}, j}^{\prime}\right\}_{j \in\{1,2,5\}}$. Then, generate a NIWI proof $\pi_{\sigma_{v_{i}}}$ that committed variables satisfy

$$
\begin{aligned}
& A^{(0)} \cdot e\left(\theta_{l, 3}^{\prime}, \theta_{l, 4}^{\prime}\right)^{-1}=e\left(G_{z}^{(0)}, \theta_{l, 1}^{\prime}\right) \cdot e\left(G_{r}^{(0)}, \theta_{l, 2}^{\prime}\right) \cdot e\left(G_{1}^{(0)}, X\right) \cdot e\left(G_{2}^{(0)}, C_{v_{i}}\right), \\
& B^{(0)} \cdot e\left(\theta_{l, 6}^{\prime}, \theta_{l, 7}^{\prime}\right)^{-1}=e\left(H_{z}^{(0)}, \theta_{l, 1}\right) \cdot e\left(H_{r}^{(0)}, \theta_{l, 5}^{\prime}\right) \cdot e\left(H_{1}^{(0)}, X\right) \cdot e\left(H_{2}^{(0)}, C_{v_{i}}\right)
\end{aligned}
$$


5. Using VK as a tag, compute a tag-based encryption 41 of $X$ by computing $\left(\Upsilon_{1}, \Upsilon_{2}, \Upsilon_{3}, \Upsilon_{4}, \Upsilon_{5}\right)=\left(f_{1}^{z_{1}}, f_{2}^{z_{2}}, X \cdot g^{z_{1}+z_{2}},\left(g^{\mathrm{VK}} \cdot U\right)^{z_{1}},\left(g^{\mathrm{VK}} \cdot V\right)^{z_{2}}\right)$ with $z_{1}, z_{2} \stackrel{R}{\leftarrow} \mathbb{Z}_{p}$.

6. Generate a NIZK proof that $\operatorname{com}_{X}=(1,1, X) \cdot{\overrightarrow{f_{1}}}^{w_{X, 1}} \cdot \vec{f}_{2}^{w_{X, 2}} \cdot \vec{f}_{3}^{w_{X, 3}}$ and $\left(\Upsilon_{1}, \Upsilon_{2}, \Upsilon_{3}\right)$ are BBS encryptions of the same value $X$. If we write $\overrightarrow{f_{3}}=\left(f_{3,1}, f_{3,2}, f_{3,3}\right)$, the Groth-Sahai commitment com $_{X}$ can be written as $\left(f_{1}^{w_{X, 1}} \cdot f_{3,1}^{w_{X, 3}}, f_{2}^{w_{X, 2}} \cdot f_{3,2}^{w_{X, 3}}, X \cdot g^{w_{X, 1}+w_{X, 2}} \cdot f_{3,3}^{w_{X, 3}}\right)$, so that we have

$$
\operatorname{com}_{X} \cdot\left(\Upsilon_{1}, \Upsilon_{2}, \Upsilon_{3}\right)^{-1}=\left(f_{1}^{\chi_{1}} \cdot f_{3,1}^{\chi_{3}}, f_{2}^{\chi_{2}} \cdot f_{3,2}^{\chi_{3}}, g^{\chi_{1}+\chi_{2}} \cdot f_{3,3}^{\chi_{3}}\right)
$$

with $\chi_{1}=w_{X, 1}-z_{1}, \chi_{2}=w_{X, 2}-z_{2}, \chi_{3}=w_{X, 3}$. To prove (10), compute $\operatorname{com}_{\chi_{j}}=\vec{\varphi}^{\chi_{j}} \cdot{\overrightarrow{f_{1}}}^{w_{\chi_{j}, 1}} \cdot{\overrightarrow{f_{2}}}^{w_{\chi_{j}, 2}}$, with $w_{\chi_{j}, 1}, w_{\chi_{j}, 2} \stackrel{R}{\leftarrow} \mathbb{Z}_{p}$ for $j \in\{1,2,3\}$, as commitments to $\left\{\chi_{j}\right\}_{j=1}^{3}$ and generates proofs $\left\{\pi_{e q-c o m, j}\right\}_{j=1}^{3}$ that $\chi_{1}, \chi_{2}, \chi_{3}$ satisfy the three linear relations (10).

7. Compute a weak Boneh-Boyen signature $\sigma_{\mathrm{VK}}=g^{1 /(x+\mathrm{VK})}$ on VK and a commitment $\operatorname{com}_{\sigma_{\mathrm{VK}}}$ to $\sigma_{\mathrm{VK}}$. Then, generate a NIWI proof $\pi_{\sigma_{\mathrm{VK}}}=$ $\left(\vec{\pi}_{\sigma_{\mathrm{VK}}, 1}, \vec{\pi}_{\sigma_{\mathrm{VK}}, 2}, \vec{\pi}_{\sigma_{\mathrm{VK}}, 3}\right) \in \mathbb{G}^{9}$ that committed variables $\left(\sigma_{\mathrm{VK}}, X\right) \in \mathbb{G}^{2}$ satisfy the quadratic equation $e\left(\sigma_{\mathrm{VK}}, X \cdot g^{\mathrm{VK}}\right)=e(g, g)$.

8. Compute $\sigma_{\text {ots }}=\mathcal{S}\left(\mathrm{SK},\left(M, R L_{t}, \Upsilon_{1}, \Upsilon_{2}, \Upsilon_{3}, \Upsilon_{4}, \Upsilon_{5}, \Omega\right.\right.$, com, $\left.\left.\mathbf{\Pi}\right)\right)$ where $\Omega=$ $\left\{\Theta_{l, i}^{\prime}, \theta_{l, i}^{\prime}\right\}_{i \in\{3,4,6,7\}}, \mathbf{\Pi}=\left(\pi_{e q}, \pi_{n e q}, \pi_{R_{l}}, \pi_{\sigma_{v_{i}}},\left\{\pi_{e q-c o m, j}\right\}_{j=1}^{3},, \pi_{\sigma_{\mathrm{VK}}}\right)$ and

$$
\begin{aligned}
& \mathbf{c o m}=\left(\operatorname{com}_{C_{v_{i}}}, \operatorname{com}_{X},\left\{\operatorname{com}_{R_{l, \tau}}\right\}_{\tau=2}^{5}, \operatorname{com}_{W_{\phi_{l}}}, \operatorname{com}_{W_{\psi_{l}}},\left\{\operatorname{com}_{\Theta_{l, j}^{\prime}}\right\}_{j \in\{1,2,5\}},\right. \\
& \left.\operatorname{com}_{\Gamma_{l}},\left\{\operatorname{com}_{\Psi_{l, \tau}}\right\}_{\tau \in\{0,1,2 \ell\}},\left\{\operatorname{com}_{\theta_{l, j}^{\prime}}\right\}_{j \in\{1,2,5\}},\left\{\operatorname{com}_{\chi_{j}}\right\}_{j=1}^{3}, \operatorname{com}_{\sigma_{\mathrm{VK}}}\right)
\end{aligned}
$$

Return the signature $\sigma=\left(\mathrm{VK}, \Upsilon_{1}, \Upsilon_{2}, \Upsilon_{3}, \Upsilon_{4}, \Upsilon_{5}, \Omega\right.$, com, $\left.\mathbf{\Pi}, \sigma_{\text {ots }}\right)$.

$\operatorname{Verify}\left(\sigma, M, t, R L_{t}, \mathcal{Y}\right)$ : If $\mathcal{V}\left(\mathrm{VK},\left(M, R L_{t}, \Upsilon_{1}, \Upsilon_{2}, \Upsilon_{3}, \Upsilon_{4}, \Upsilon_{5}, \Omega, \mathbf{c o m}, \mathbf{\Pi}\right), \sigma_{\text {ots }}\right)=$ 0 or if $\left(\Upsilon_{1}, \Upsilon_{2}, \Upsilon_{3}, \Upsilon_{4}, \Upsilon_{5}\right)$ is not a well-formed tag-based encryption (that is, if $e\left(\Upsilon_{1}, g^{\mathrm{VK}} \cdot U\right) \neq e\left(f_{1}, \Upsilon_{4}\right)$ or $\left.e\left(\Upsilon_{2}, g^{\mathrm{VK}} \cdot V\right) \neq e\left(f_{2}, \Upsilon_{5}\right)\right)$, return 0 . Then, return 1 if all proofs properly verify. Otherwise, return 0.

$\operatorname{Open}\left(M, t, R L_{t}, \sigma, \mathcal{S}_{\mathrm{OA}}, \mathcal{Y}, S t\right)$ : Return $\perp$ if $\operatorname{Verify}\left(\sigma, M, t, R L_{t}, \mathcal{Y}\right)=0$. Otherwise, given $\mathcal{S}_{\mathrm{OA}}=\left(\beta_{1}, \beta_{2}\right)$, compute $\tilde{X}=\Upsilon_{3} \cdot \Upsilon_{1}^{-1 / \beta_{1}} \cdot \Upsilon_{2}^{-1 / \beta_{2}}$. In the database $S t_{\text {trans }}$, find a record $\left\langle i\right.$, transcript $\left.{ }_{i}=\left(X_{i}, \operatorname{ID}\left(v_{i}\right), C_{v_{i}}, \sigma_{v_{i}}, s i g_{i}\right)\right\rangle$ such that $X_{i}=\tilde{X}$. If no such record exists in $S t_{\text {trans }}$, return $\perp$. Otherwise, return $i$.

At first glance, the variable $\Psi_{l, 2 \ell}$ and the proof of the second equality (8) may seem unnecessary in step 2.b of the signing algorithm. However, as detailed in the full version of the paper, this element plays a crucial role when it comes to prove the security under the $\ell$-FlexDHE assumption.

As far as efficiency goes, each entry of $R L_{t}$ contains 7 group elements and two node identifiers of $O(\log N)$ bits each. If $\lambda_{\mathbb{G}}$ is the bitlength of a group element, we have $\log N \ll \lambda_{\mathbb{G}} / 2$ (since $\lambda \leq \lambda_{\mathbb{G}}$ and $N$ is polynomial), so that the number of bits of $R L_{t}$ is bounded by $2 \cdot\left|\mathcal{R}_{t}\right| \cdot\left(7 \cdot \lambda_{\mathbb{G}}+2 \log N+2 \log \log N\right)<2 \cdot\left|\mathcal{R}_{t}\right| \cdot\left(9 \lambda_{\mathbb{G}}\right)$ bits. The size of $R L_{t}$ is thus bounded by that of $18 \cdot\left|\mathcal{R}_{t}\right|$ group elements.

Unlike 44, group members only need to store 9 group elements in their membership certificate. As far as the size of signature goes, com and $\boldsymbol{\Pi}$ require 
66 and 60 group elements, respectively. If the one-time signature of [34] is used, VK and $\sigma_{\text {ots }}$ consist of 3 elements of $\mathbb{G}$ and 2 elements of $\mathbb{Z}_{p}$, respectively. The global size $\sigma$ amounts to that of 144 group elements, which is about $50 \%$ longer than [4]. In comparison with [35] (which does not natively support revocation), signatures are only longer by a factor of 3 . At the 128-bit security level, each group element should have a 512-bit representation and a signature takes $9 \mathrm{kB}$.

Verifying signatures takes constant time. The signer has to compute at most $2 \ell=O(\log N)$ exponentiations to obtain $W_{\phi_{l}}$ and $W_{\psi_{l}}$ at the beginning of each revocation epoch. Note that these exponentiations involve short exponents of $O(\log N)$ bits each. Hence, computing $W_{\phi_{l}}$ and $W_{\psi_{l}}$ requires $O\left(\log ^{2} N\right)$ multiplications in $\mathbb{G}$. For this reason, since we always have $\log ^{2} N \ll \lambda$ (as long as $N \ll 2^{\lambda^{1 / 2}}$ ), this cost is dominated by that of a single exponentiation in $\mathbb{G}$.

From a security point of view, we prove the following theorem in the full version of the paper.

Theorem 1. Under the SFP, FlexDHE, SDH and DLIN assumptions, the scheme provides anonymity and security against misidentification and framing attacks .

\section{References}

1. Abe, M., Haralambiev, K., Ohkubo, M.: Signing on Elements in Bilinear Groups for Modular Protocol Design. Cryptology ePrint Archive: Report 2010/133 (2010)

2. Abe, M., Fuchsbauer, G., Groth, J., Haralambiev, K., Ohkubo, M.: StructurePreserving Signatures and Commitments to Group Elements. In: Rabin, T. (ed.) CRYPTO 2010. LNCS, vol. 6223, pp. 209-236. Springer, Heidelberg (2010)

3. Abe, M., Groth, J., Haralambiev, K., Ohkubo, M.: Optimal Structure-Preserving Signatures in Asymmetric Bilinear Groups. In: Rogaway, P. (ed.) CRYPTO 2011. LNCS, vol. 6841, pp. 649-666. Springer, Heidelberg (2011)

4. Acar, T., Nguyen, L.: Revocation for Delegatable Anonymous Credentials. In: Catalano, D., Fazio, N., Gennaro, R., Nicolosi, A. (eds.) PKC 2011. LNCS, vol. 6571, pp. 423-440. Springer, Heidelberg (2011)

5. Ateniese, G., Camenisch, J., Hohenberger, S., de Medeiros, B.: Practical group signatures without random oracles. Cryptology ePrint Archive: Report 2005/385 (2005)

6. Ateniese, G., Camenisch, J., Joye, M., Tsudik, G.: A Practical and Provably Secure Coalition-Resistant Group Signature Scheme. In: Bellare, M. (ed.) CRYPTO 2000. LNCS, vol. 1880, pp. 255-270. Springer, Heidelberg (2000)

7. Ateniese, G., Song, D., Tsudik, G.: Quasi-Efficient Revocation in Group Signatures. In: Blaze, M. (ed.) FC 2002. LNCS, vol. 2357, pp. 183-197. Springer, Heidelberg (2003)

8. Bellare, M., Micciancio, D., Warinschi, B.: Foundations of Group Signatures: Formal Definitions, Simplified Requirements, and a Construction Based on General Assumptions. In: Biham, E. (ed.) EUROCRYPT 2003. LNCS, vol. 2656, pp. 614629. Springer, Heidelberg (2003)

9. Bellare, M., Rogaway, P.: Random Oracles are Practical: A Paradigm for Designing Efficient Protocols. In: 1st ACM Conference on Computer and Communications Security, pp. 62-73. ACM Press (1993) 
10. Bellare, M., Shi, H., Zhang, C.: Foundations of Group Signatures: The Case of Dynamic Groups. In: Menezes, A. (ed.) CT-RSA 2005. LNCS, vol. 3376, pp. 136153. Springer, Heidelberg (2005)

11. Benaloh, J., de Mare, M.: One-Way Accumulators: A Decentralized Alternative to Digital Signatures. In: Helleseth, T. (ed.) EUROCRYPT 1993. LNCS, vol. 765, pp. 274-285. Springer, Heidelberg (1994)

12. Boneh, D., Boyen, X.: Short Signatures Without Random Oracles. In: Cachin, C., Camenisch, J. (eds.) EUROCRYPT 2004. LNCS, vol. 3027, pp. 56-73. Springer, Heidelberg (2004)

13. Boneh, D., Boyen, X., Goh, E.-J.: Hierarchical Identity Based Encryption with Constant Size Ciphertext. In: Cramer, R. (ed.) EUROCRYPT 2005. LNCS, vol. 3494, pp. 440-456. Springer, Heidelberg (2005)

14. Boneh, D., Boyen, X., Shacham, H.: Short Group Signatures. In: Franklin, M. (ed.) CRYPTO 2004. LNCS, vol. 3152, pp. 41-55. Springer, Heidelberg (2004)

15. Boneh, D., Franklin, M.: Identity based encryption from the Weil pairing. SIAM J. of Computing 32(3), 586-615 (2003)

16. Boneh, D., Gentry, C., Waters, B.: Collusion Resistant Broadcast Encryption with Short Ciphertexts and Private Keys. In: Shoup, V. (ed.) CRYPTO 2005. LNCS, vol. 3621, pp. 258-275. Springer, Heidelberg (2005)

17. Boneh, D., Shacham, H.: Group signatures with verifier-local revocation. In: ACMCCS 2004, pp. 168-177. ACM Press (2004)

18. Boyen, X., Waters, B.: Compact Group Signatures Without Random Oracles. In: Vaudenay, S. (ed.) EUROCRYPT 2006. LNCS, vol. 4004, pp. 427-444. Springer, Heidelberg (2006)

19. Boyen, X., Waters, B.: Full-Domain Subgroup Hiding and Constant-Size Group Signatures. In: Okamoto, T., Wang, X. (eds.) PKC 2007. LNCS, vol. 4450, pp. 1-15. Springer, Heidelberg (2007)

20. Bresson, E., Stern, J.: Efficient Revocation in Group Signatures. In: Kim, K.-C. (ed.) PKC 2001. LNCS, vol. 1992, pp. 190-206. Springer, Heidelberg (2001)

21. Brickell, E.: An efficient protocol for anonymously providing assurance of the container of the private key. Submission to the Trusted Computing Group (April 2003)

22. Brickell, E., Camenisch, J., Chen, L.: Direct Anonymous Attestation. In: ACMCCS 2004, pp. 132-145 (2004)

23. Camenisch, J., Chaabouni, R., Shelat, A.: Efficient Protocols for Set Membership and Range Proofs. In: Pieprzyk, J. (ed.) ASIACRYPT 2008. LNCS, vol. 5350, pp. 234-252. Springer, Heidelberg (2008)

24. Camenisch, J., Kohlweiss, M., Soriente, C.: An Accumulator Based on Bilinear Maps and Efficient Revocation for Anonymous Credentials. In: Jarecki, S., Tsudik, G. (eds.) PKC 2009. LNCS, vol. 5443, pp. 481-500. Springer, Heidelberg (2009)

25. Camenisch, J., Kohlweiss, M., Soriente, C.: Solving Revocation with Efficient Update of Anonymous Credentials. In: Garay, J.A., De Prisco, R. (eds.) SCN 2010. LNCS, vol. 6280, pp. 454-471. Springer, Heidelberg (2010)

26. Camenisch, J., Lysyanskaya, A.: Dynamic Accumulators and Application to Efficient Revocation of Anonymous Credentials. In: Yung, M. (ed.) CRYPTO 2002. LNCS, vol. 2442, pp. 61-76. Springer, Heidelberg (2002)

27. Catalano, D., Fiore, D.: Concise Vector Commitments and their Applications to Zero-Knowledge Elementary Databases. Cryptology ePrint Archive: Report 2011/495 (2011)

28. Cathalo, J., Libert, B., Yung, M.: Group Encryption: Non-interactive Realization in the Standard Model. In: Matsui, M. (ed.) ASIACRYPT 2009. LNCS, vol. 5912, pp. 179-196. Springer, Heidelberg (2009) 
29. Chaum, D., van Heyst, E.: Group Signatures. In: Davies, D.W. (ed.) EUROCRYPT 1991. LNCS, vol. 547, pp. 257-265. Springer, Heidelberg (1991)

30. Delerablée, C., Pointcheval, D.: Dynamic Fully Anonymous Short Group Signatures. In: Nguyên, P.Q. (ed.) VIETCRYPT 2006. LNCS, vol. 4341, pp. 193-210. Springer, Heidelberg (2006)

31. Dodis, Y., Fazio, N.: Public Key Broadcast Encryption for Stateless Receivers. In: Feigenbaum, J. (ed.) DRM 2002. LNCS, vol. 2696, pp. 61-80. Springer, Heidelberg (2003)

32. Fuchsbauer, G.: Automorphic Signatures in Bilinear Groups and an Application to Round-Optimal Blind Signatures. Cryptology ePrint Archive: Report 2009/320 (2009)

33. Gentry, C., Silverberg, A.: Hierarchical ID-Based Cryptography. In: Zheng, Y. (ed.) ASIACRYPT 2002. LNCS, vol. 2501, pp. 548-566. Springer, Heidelberg (2002)

34. Groth, J.: Simulation-Sound NIZK Proofs for a Practical Language and Constant Size Group Signatures. In: Lai, X., Chen, K. (eds.) ASIACRYPT 2006. LNCS, vol. 4284, pp. 444-459. Springer, Heidelberg (2006)

35. Groth, J.: Fully Anonymous Group Signatures Without Random Oracles. In: Kurosawa, K. (ed.) ASIACRYPT 2007. LNCS, vol. 4833, pp. 164-180. Springer, Heidelberg (2007)

36. Groth, J., Sahai, A.: Efficient Non-interactive Proof Systems for Bilinear Groups. In: Smart, N.P. (ed.) EUROCRYPT 2008. LNCS, vol. 4965, pp. 415-432. Springer, Heidelberg (2008)

37. Halevy, D., Shamir, A.: The LSD Broadcast Encryption Scheme. In: Yung, M. (ed.) CRYPTO 2002. LNCS, vol. 2442, pp. 47-60. Springer, Heidelberg (2002)

38. Horwitz, J., Lynn, B.: Toward Hierarchical Identity-Based Encryption. In: Knudsen, L.R. (ed.) EUROCRYPT 2002. LNCS, vol. 2332, pp. 466-481. Springer, Heidelberg (2002)

39. Izabachène, M., Libert, B., Vergnaud, D.: Block-Wise P-Signatures and Noninteractive Anonymous Credentials with Efficient Attributes. In: Chen, L. (ed.) IMACC 2011. LNCS, vol. 7089, pp. 431-450. Springer, Heidelberg (2011)

40. Kiayias, A., Yung, M.: Secure scalable group signature with dynamic joins and separable authorities. International Journal of Security and Networks (IJSN) 1(1/2), 24-45 (2006)

41. Kiltz, E.: Chosen-Ciphertext Security from Tag-Based Encryption. In: Halevi, S., Rabin, T. (eds.) TCC 2006. LNCS, vol. 3876, pp. 581-600. Springer, Heidelberg (2006)

42. Libert, B., Vergnaud, D.: Group Signatures with Verifier-Local Revocation and Backward Unlinkability in the Standard Model. In: Garay, J.A., Miyaji, A., Otsuka, A. (eds.) CANS 2009. LNCS, vol. 5888, pp. 498-517. Springer, Heidelberg (2009)

43. Libert, B., Yung, M.: Concise Mercurial Vector Commitments and Independent Zero-Knowledge Sets with Short Proofs. In: Micciancio, D. (ed.) TCC 2010. LNCS, vol. 5978, pp. 499-517. Springer, Heidelberg (2010)

44. Libert, B., Peters, T., Yung, M.: Scalable Group Signatures with Revocation. In: Pointcheval, D., Johansson, T. (eds.) EUROCRYPT 2012. LNCS, vol. 7237, pp. 609-627. Springer, Heidelberg (2012)

45. Nakanishi, T., Fujii, H., Hira, Y., Funabiki, N.: Revocable Group Signature Schemes with Constant Costs for Signing and Verifying. In: Jarecki, S., Tsudik, G. (eds.) PKC 2009. LNCS, vol. 5443, pp. 463-480. Springer, Heidelberg (2009)

46. Nakanishi, T., Funabiki, N.: Verifier-Local Revocation Group Signature Schemes with Backward Unlinkability from Bilinear Maps. In: Roy, B. (ed.) ASIACRYPT 2005. LNCS, vol. 3788, pp. 533-548. Springer, Heidelberg (2005) 
47. Naor, D., Naor, M., Lotspiech, J.: Revocation and Tracing Schemes for Stateless Receivers. In: Kilian, J. (ed.) CRYPTO 2001. LNCS, vol. 2139, pp. 41-62. Springer, Heidelberg (2001)

48. Nguyen, L.: Accumulators from Bilinear Pairings and Applications. In: Menezes, A. (ed.) CT-RSA 2005. LNCS, vol. 3376, pp. 275-292. Springer, Heidelberg (2005)

49. Nguyen, L., Safavi-Naini, R.: Efficient and Provably Secure Trapdoor-Free Group Signature Schemes from Bilinear Pairings. In: Lee, P.J. (ed.) ASIACRYPT 2004. LNCS, vol. 3329, pp. 372-386. Springer, Heidelberg (2004)

50. Song, D.: Practical forward secure group signature schemes. In: ACM-CCS 2001, pp. 225-234 (2001)

51. Tsang, P., Au, M.-H., Kapadia, A., Smith, S.: Blacklistable anonymous credentials: blocking misbehaving users without TTPs. In: ACM-CCS 2007, pp. 72-81 (2007)

52. Tsudik, G., Xu, S.: Accumulating Composites and Improved Group Signing. In: Laih, C.-S. (ed.) ASIACRYPT 2003. LNCS, vol. 2894, pp. 269-286. Springer, Heidelberg (2003)

53. Zhou, S., Lin, D.: Shorter Verifier-Local Revocation Group Signatures from Bilinear Maps. In: Pointcheval, D., Mu, Y., Chen, K. (eds.) CANS 2006. LNCS, vol. 4301, pp. 126-143. Springer, Heidelberg (2006) 